\title{
Some Elastic Properties and Phase Transitions in Some Alkali Halides Using Interatomic Potential Model
}

\author{
H.R. YAZAR \\ Department of Physics, Kırıkkale University \\ 71450, Yahşihan, Kırıkkale, Turkey \\ (Received April 26, 2005; revised version November 14, 2005; \\ in final form January 30, 2006)
}

\begin{abstract}
We have predicted the phase transition pressure in some alkali halides using an interatomic potential approach based on rigid ion model. This potential form represents the composite form of the inverse power dependence and exponential dependence of the repulsive energy on interionic distance. Some thermoelastic properties such as second order elastic constants have been calculated using the same potential. The phase transition pressures (28.69 and 2.4 GPa) obtained by us for two alkali halides ( $\mathrm{NaCl}$ and $\mathrm{KCl})$ are in closer agreement with their corresponding experimental data (29.0 and 2.0 GPa). This approach is promising with respect to prediction of the phase transition pressure of other alkali halides as well.
\end{abstract}

PACS numbers: 78.55.Fv, 05.70.--a, 61.50.Ks, 64.10.+h

\section{Introduction}

The quantum mechanical calculation of the repulsive energy of a pair of multielectron atoms or ions is an extremely difficult and complicated task even for simple condensed systems of closed shell ions such as NaCl-like alkali halides. Semiemprical and phenomenological potential forms have therefore been developed which represent the exponential dependence on interionic distance such as the Born-Mayer exponential form, and the inverse power dependence as the BornLande inverse power form. Studies on the alkali halides provide a critical test of different interionic potentials.

The alkali halides are the simplest and ideal ionic solids on which much experimental and theoretical work has been done in the past [1]. They are model crystals for performing tests to validate new theories. They generally crystallize in either the $\mathrm{NaCl}(\mathrm{B} 1)$ or the $\mathrm{CsCl}(\mathrm{B} 2)$ structure. Their elastic, dynamic, and thermodynamic properties have been extensively investigated by various experimental and theoretical workers $[2,3]$. These solids undergo structural phase transition $(\mathrm{B} 1 \rightarrow \mathrm{B} 2)$ at elevated pressures [4]. A survey of the literature reveals that, 
although a large amount of experimental work has been done on the phase transition in alkali halides [5], very scant attention has been paid to their theoretical understanding.

The effects of such a non-rigidity of ions via three-body interaction have been incorporated in the successful study of the phase transition and high-pressure elastic behavior of II-VI and III-V compound semiconductors [6] and divalent metal oxides [7]. Motivated by these facts, we thought it pertinent to apply the interatomic potential for the prediction of the phase transition pressures in $\mathrm{NaCl}$ and $\mathrm{KCl}$. In the present paper we proposed an interatomic potential and we investigate the applicability of an interatomic potential by calculating second order elastic constants (SOEC) and some physical quantities such as the phase transition pressures of two alkali halides $(\mathrm{NaCl}$ and $\mathrm{KCl})$.

In this paper, the three-body interaction has been considered to arise from the effects of non-rigidity of ions caused by the deformation of the electron shells of the overlapping ions. We have adopted the graphical method for predicting the phase transition pressure by plotting the Gibbs free energy as a function of pressure.

\section{Theory and method of calculation}

The lattice energy for an ionic crystal in terms of an effective pair potential is written as follows [8]:

$$
\begin{gathered}
\varphi\left(r_{i j} r_{i k}\right)=-\sum_{j \neq k} \sum_{i} \frac{\alpha_{\mathrm{M}} e^{2}}{r_{i j}+r_{i k}}+\frac{V_{i j}}{\left(r_{i j}+r_{i k}\right)^{n}} \\
-\frac{C}{\left(r_{i j}+r_{i k}\right)^{6}}-\frac{D}{\left(r_{i j}+r_{i k}\right)^{8}},
\end{gathered}
$$

where $V_{i j}=\beta_{i j} b \exp \left(-\left(r_{i j}+r_{i k}\right)^{m} / \rho_{i j}\right)$ is the short-range potential. The repulsive parameters $b$ and $\rho$ which are the values of adjustable model parameters for the different salts are given in Table I. $r_{i j}$ is the interatomic distance between atoms $i$ - and $j$-th and $r_{0}$ is the separation of the atoms for minimum potential. The three-body general potential coupling the atom $i$-th with its neighbor's $j$ - and $k$-th has been defined in terms of the two-body model potential. The first term on the right hand side of Eq. (1) is the long-range electrostatic Madelung energy term $\alpha_{\mathrm{M}}$, the second term represents the short-range overlap repulsive interaction $V_{i j}$, and the last two terms represent the Van Der Waals dipole-dipole $\left(C_{\mathrm{NaCl}}=\right.$ $180 \times 10^{-60} \mathrm{erg} / \mathrm{mole}$ and $\left.C_{\mathrm{KCl}}=452 \times 10^{-60} \mathrm{erg} / \mathrm{mole}\right)$ and dipole-quadrupole $\left(D_{\mathrm{NaCl}}=180 \times 10^{-76} \mathrm{erg} / \mathrm{mole}\right.$ and $\left.D_{\mathrm{KCl}}=560 \times 10^{-76} \mathrm{erg} / \mathrm{mole}\right)$ interactions, respectively [3]. Following Michielsen et al. [9], we take $n=4$ and $m=1$ so that

$$
\begin{gathered}
\varphi\left(r_{i j} r_{i k}\right)=-\sum_{j \neq k} \sum_{i} \frac{\alpha_{\mathrm{M}} e^{2}}{r_{i j}+r_{i k}}+\frac{\beta_{i j} b}{\left(r_{i j}+r_{i k}\right)^{4}} \exp \left(-\frac{\left(r_{i j}+r_{i k}\right)^{1}}{\rho_{i j}}\right) \\
-\frac{C}{\left(r_{i j}+r_{i k}\right)^{6}}-\frac{D}{\left(r_{i j}+r_{i k}\right)^{8}} .
\end{gathered}
$$


The equilibrium condition is [1]

$$
\begin{gathered}
\left(b / r_{0}\right)\left[\beta_{i j} \exp \left(\left(r_{i}+r_{j}-r_{0}\right) / \rho_{i j}\right)+2 \beta_{i j} \exp \left(\left(2 r_{i}-\sqrt{2} r_{0}\right) / \rho_{i j}\right)\right. \\
\left.+2 \beta_{i j} \exp \left(\left(2 r_{j}-\sqrt{2} r_{0}\right) / \rho_{i j}\right)\right]=\left(2 \alpha_{\mathrm{M}} / 3\right) z^{2} e^{2} / 2 v,
\end{gathered}
$$

where $\beta_{i j}(i, j=1,2)$ are the Pauling coefficients, $b$ and $\rho$ are the range parameters, $v$ is the unit cell volume. For simplicity we have taken single $\rho$ instead of the $\rho_{i j}$.

This potential has only two parameters $b$ and $\rho$ and can be determined from the equilibrium condition using the value of the lattice constants [10]. The calculated values of these parameters $(b, \rho)$ and input parameters, such as $r_{0}, r_{+}, r_{-}, B_{0}$ which were taken from literature [10], are listed in Table I and used to compute the phase transition pressure and volume collapses in $\mathrm{NaCl}$ and $\mathrm{KCl}$ at $0 \mathrm{~K}$.

\section{TABLE I}

Input data and model parameters of $\mathrm{NaCl}$ and KCI.

\begin{tabular}{c|c|c|c|c|c|c|c}
\hline \hline & $r_{0}$ & $r_{+}$ & $r_{-}$ & $B_{0}$ & $f_{0}$ & \multicolumn{2}{|c}{ Model parameters } \\
\hline & & & & & & $b\left[10^{-12} \mathrm{erg}\right]$ & $\rho[\AA]$ \\
\cline { 5 - 7 } $\mathrm{NaCl}$ & 2.82 & 1.54 & 1.99 & 240 & 8.78 & 0.341 & 0.353 \\
$\mathrm{KCl}$ & 3.14 & 1.51 & 1.63 & 173 & 32.547 & 0.313 & 0.315 \\
\hline
\end{tabular}

The $r_{0}, r_{+}, r_{-}$are in $\AA$ units and $B_{0}$ (bulk modulus) is in $10^{8} \mathrm{~Pa}[10]$.

Thermodynamically, a phase transition is said to occur when the changes in structural parameters of the phase are caused by the variation of free energy. The compound transform from its initial $\mathrm{B} 1(\mathrm{NaCl})$ to $\mathrm{B} 2(\mathrm{CsCl})$ structure under pressure. The difference between free energy of the two phases becomes zero at the phase transition pressure. The stability of a particular structure is determined by the minimum of the Gibbs energy which is

$$
G=U+P V-T S,
$$

where $U$ is the internal energy, which at $0 \mathrm{~K}$ corresponds to cohesive energy, $S$ is the vibrational entropy at absolute $T$, pressure $P$, and volume $V$.

The Gibbs free energies $G_{\mathrm{B} 1}(r)=U_{\mathrm{B} 1}(r)+2 P r^{3}$ for the $\mathrm{NaCl}(\mathrm{B} 1)$ phase and $G_{\mathrm{B} 2}\left(r^{\prime}\right)=U_{\mathrm{B} 2}\left(r^{\prime}\right)+8 \sqrt{3} /\left(3 \operatorname{Pr}^{\prime 3}\right)$ for the $\mathrm{CsCl}(\mathrm{B} 2)$ phase become equal at the phase transition pressure $P$ and temperature 0 K, i.e. $\Delta G=\left(G_{\mathrm{B} 1}-G_{\mathrm{B} 2}\right)$ becomes zero. $U_{\mathrm{B} 1}$ and $U_{\mathrm{B} 2}$ phases are given by [1]

$$
U_{\mathrm{B} 1}(r)=-1.7475 e^{2} Z(Z+6 f(r)) / r+6 V_{i j}(r)+6 V_{i i}(r)+6 V_{j j}(r)
$$

and

$$
U_{\mathrm{B} 2}\left(r^{\prime}\right)=-1.7627 e^{2} Z\left(Z+8 f\left(r^{\prime}\right)\right) / r^{\prime}+8 V_{i j}\left(r^{\prime}\right)+8 V_{i i}\left(r^{\prime}\right)+8 V_{j j}\left(r^{\prime}\right) .
$$

Respectively, here $r$ and $r^{\prime}$ are nearest neighbor (nn) separations corresponding to $\mathrm{NaCl}$ and $\mathrm{CsCl}$ phases, respectively. $f(r)$ and $f\left(r^{\prime}\right)$ are the two-body force parameters for $\mathrm{NaCl}$ and $\mathrm{CsCl}$, respectively, expressed as [11] 


$$
f(r)=f_{0} \exp \left(-r / \rho_{i j}\right), \quad f\left(r^{\prime}\right)=f_{0} \exp \left(-r^{\prime} / \rho_{i j}\right)
$$

Here, the input values of $f_{0}$ are taken to be the same for both structures $(\mathrm{NaCl}$ and $\mathrm{CsCl}$ ) [12].

For predicting transition pressure, we have minimized the Gibbs free energies with respect to interatomic separations and calculated $\Delta G=\left(G_{\mathrm{B} 1}-G_{\mathrm{B} 2}\right)$ for various pressures. The pressure, at which $\Delta G$ approaches zero, corresponds to the phase transition.

\subsection{Elastic constants}

The derivative of the potential energy $\varphi$ for central forces is most conveniently expressed in spherical coordinates. The transformation from rectangular to spherical coordinates is given by [13]

$$
\begin{aligned}
& \left(\frac{\partial \varphi}{\partial \alpha}\right)_{r}=\alpha \boldsymbol{P} \\
& \left(\frac{\partial^{2} \varphi}{\partial \alpha \partial \beta}\right)_{r}=\delta_{\alpha \beta} \boldsymbol{P}+\alpha \beta \boldsymbol{Q},
\end{aligned}
$$

where $\boldsymbol{P}$ and $\boldsymbol{Q}$ are the derivative operators as follows,

$$
\begin{aligned}
& \boldsymbol{P}=\left(\frac{1}{r} \frac{\partial \varphi}{\partial r}\right), \\
& \boldsymbol{Q}=\left[\frac{1}{r} \frac{\partial}{\partial r}\left(\frac{1}{r} \frac{\partial \varphi}{\partial r}\right)\right] .
\end{aligned}
$$

$\beta \equiv \frac{1}{V}\left(\frac{\partial V}{\partial T}\right)_{P}$ and $\alpha \equiv \frac{1}{P}\left(\frac{\partial P}{\partial T}\right)_{V}$ are mechanical coefficients of related materials,

$$
\delta_{\alpha \beta}= \begin{cases}1 & \text { for } \alpha=\beta \\ 0 & \text { for } \alpha \neq \beta\end{cases}
$$

The equations for the elastic constants are then defined by Anderson [14], where $\Delta$ is the volume-per cell (ion pair):

$$
\begin{aligned}
& C_{11}=\left(C_{x x y x}\right)=\frac{1}{\Delta} \sum_{l}\left[\boldsymbol{Q}\left(x^{l}\right)^{4}+\boldsymbol{P}\left(x^{l}\right)^{2}\right], \\
& C_{12}=\left(C_{x x y y}\right)=\frac{1}{\Delta} \sum_{l}\left[\boldsymbol{Q}\left(x^{l}\right)^{2}\left(y^{l}\right)^{2}-\boldsymbol{P}\left(x^{l}\right)^{2}\right], \\
& C_{44}=\left(C_{x y x y}^{*}\right)=\frac{1}{\Delta} \sum_{l}\left[\boldsymbol{Q}\left(x^{l}\right)^{2}\left(y^{l}\right)^{2}+\boldsymbol{P}\left(x^{l}\right)^{2}\right] .
\end{aligned}
$$

SOEC have been obtained from transportation of Cartesian coordinates to spherical coordinates system and given in Table II. Since forces are additive the derivative of the potential in the equations of motion are additive, and the elastic constants can be broken down into a sum

$$
\left(C_{\alpha \beta \gamma \lambda}\right)=\left(C_{\alpha \beta \gamma \lambda}\right)^{e}+\left(C_{\alpha \beta \gamma \lambda}\right)^{R} .
$$

The reason, that these constants are broken down into two parts, is that the 
sum motion is taken to infinity for the $e$ component (standing for electrostatic or Coulombic), while the summation is taken only over nearest neighbors for $R$ component (standing for repulsive). This separation corresponds to the separate parts of the lattice potential arising between the $k$ and $k^{\prime}$ positions which is appropriate to define the energy per unit cell

$$
\varphi_{k k^{\prime}}=-\frac{A_{r} Z_{k} Z_{k^{\prime}}^{2}}{r_{k k^{\prime}}}+M_{k} \varphi^{R}(r)
$$

where $M_{k}$ is the coordination number around the $k$ position, for the case of $\mathrm{NaCl}$, $M_{k}=6 ; A_{r}$ is Madelung constant of the lattice, $Z_{k}$ and $Z_{k^{\prime}}$ are the valence number and $e$ is the electronic charge and $\varphi^{R}(r)$ is the repulsive potential.

TABLE II

Second order elastic constants for NaCl-structure (for details see the text).

\begin{tabular}{c|c}
\hline \hline$\Delta C_{11}^{R}$ & $2 \boldsymbol{Q}^{R} r^{4}+2 \boldsymbol{P}^{R} r^{2}$ \\
$\Delta C_{12}^{R}$ & $-2 \boldsymbol{P}^{R} r^{2}$ \\
$\Delta C_{44}^{R}$ & $2 \boldsymbol{P}^{R} r^{2}$
\end{tabular}

Denoting the potential above by the symbols $R$ and $e$ corresponding to the two terms in Eq. (15), we write

$$
\varphi=\varphi^{e}+\varphi^{R}
$$

Also denote the operators $\boldsymbol{P}$ and $\boldsymbol{Q}$ by the same symbols so that

$$
\boldsymbol{P}^{R}=\frac{1}{r}\left(\frac{\partial \varphi^{R}}{\partial r}\right)_{r}, \quad \boldsymbol{Q}^{R}=\left[\frac{1}{r} \frac{\partial}{\partial r}\left(\frac{1}{r} \frac{\partial \varphi^{R}}{\partial r}\right)\right]_{r},
$$

as also the elastic constants (for example $C_{11}$ )

$$
C_{11}=C_{11}^{e}+C_{11}^{R}
$$

Repulsive parts of the elastic constant (for example $C_{11}^{R}$ ) can be expressed in terms of $\boldsymbol{P}^{R}$ and $\boldsymbol{Q}^{R}$ operators and are given in Table II. It is clearly seen from Eq. (17) that $\boldsymbol{P}^{R}$ and $\boldsymbol{Q}^{R}$ can be written as a first and second derivative of the potential and these forms are given in Table III. Madelung constants can be defined in two ways; a constant $A_{r}$ corresponding to the nearest-neighbor distance $r$, or

TABLE III

The elastic constant parameters in terms of the derivatives of the repulsive potential $\left[\varphi^{R}(r)\right]$.

\begin{tabular}{c|c}
\hline \hline$\Delta C_{11}^{R}$ & $2 r^{2} \mathrm{~d}^{2} \varphi^{R}(r) / \mathrm{d} r^{2}$ \\
$\Delta C_{12}^{R}$ & $-2 r\left(\mathrm{~d} \varphi^{R}(r) / \mathrm{d} r\right)$ \\
$\Delta C_{44}^{R}$ & $2 r\left(\mathrm{~d} \varphi^{R}(r) / \mathrm{d} r\right)$
\end{tabular}


$A_{a}$ corresponding to half distance $a$ of the unit cell. For comparison purposes, $A_{r}=A_{a}$ and similarly, $r=a$ for $\mathrm{NaCl}$.

\subsection{The short-range component of the potential}

We now express the operators $\boldsymbol{P}$ and $\boldsymbol{Q}$ in terms of the derivatives of the repulsive potential with respect to $r$. It is readily seen that

$$
\begin{aligned}
\boldsymbol{Q}^{R} & =\frac{1}{r^{2}} \frac{\mathrm{d}^{2} \varphi^{R}}{\mathrm{~d} r^{2}}-\frac{1}{r^{3}} \frac{\mathrm{d} \varphi^{R}}{\mathrm{~d} r}, \\
\boldsymbol{P}^{R} & =\frac{1}{r} \frac{\mathrm{d} \varphi^{R}}{\mathrm{~d} r} .
\end{aligned}
$$

In a tabular form, the results are given in Table III.

There are two ways to proceed from this point. One way is to represent the operators $\mathrm{d}^{2} \varphi^{R} / \mathrm{d} r^{2}$ and $\mathrm{d} \varphi^{R} / \mathrm{d} r$ at the equilibrium condition $r=r_{0}$ as pure numbers and then evaluate the numbers from experiments on the elastic constants. The second way is to assume some function through the operators. We will proceed with elastic constants as a function of $r$.

Let us take the repulsive part of the potential as

$$
\varphi^{R}(r)=\frac{b}{r^{4}} \exp \left(-\frac{r}{\rho}\right)
$$

Using Eq. (21) to the elastic parameters listed in Table III, we have evaluated the repulsive term in the elastic constants, which are given in Table IV.

The derivative of Eq. (15) is vanishing at equilibrium, $r=r_{0}$. Thus

$$
\frac{4 B \exp \left(-\frac{r}{\rho}\right)}{r^{5}}+\frac{B \exp \left(-\frac{r}{\rho}\right)}{\rho r^{4}}=\frac{A_{r} Z^{2} e^{2}}{M r^{2}}
$$

and

$$
\begin{aligned}
\boldsymbol{P}^{R} & =-\frac{B \exp \left(-\frac{r}{\rho}\right)}{\rho r^{5}}-\frac{4 B \exp \left(-\frac{r}{\rho}\right)}{r^{6}}, \\
\boldsymbol{Q}^{R} & =\frac{24 B \exp \left(-\frac{r}{\rho}\right)}{\rho r^{8}}+\frac{9 B \exp \left(-\frac{r}{\rho}\right)}{\rho r^{7}}+\frac{B \exp \left(-\frac{r}{\rho}\right)}{\rho^{2} r^{6}} .
\end{aligned}
$$

The elastic constants expressions can be obtained by combining the results shown in Table $\mathrm{V}$ with those in Table IV. The full expressions for the elastic constants for $\mathrm{NaCl}$-structure are:

$$
\begin{aligned}
& C_{11}=\frac{1}{2 M} \frac{A_{r} Z^{2} e^{2} \rho}{(4 \rho+r)}\left(\frac{40 \rho^{2}+7 \rho r+r^{2}}{\rho^{2} r^{4}}\right)-2.55604 \frac{Z^{2} e^{2}}{\Delta_{0} a_{0}}, \\
& C_{12}=\frac{A_{r} Z^{2} e^{2}}{r^{3} M(4 \rho+r)}+\frac{4 A_{r} Z^{2} e^{2} \rho}{r^{4} M(4 \rho+r)}+0.11298 \frac{Z^{2} e^{2}}{\Delta_{0} a_{0}}, \\
& C_{44}=-\frac{A_{r} Z^{2} e^{2}}{r^{3} M(4 \rho+r)}-\frac{4 A_{r} Z^{2} e^{2} \rho}{r^{4} M(4 \rho+r)}+1.27802 \frac{Z^{2} e^{2}}{\Delta_{0} a_{0}},
\end{aligned}
$$

The calculated values of SOEC and experimental values [12] of alkali halides are given in Table VI. 
TABLE IV

The elastic constant parameters for $\mathrm{NaCl}$-structure (see the text).

\begin{tabular}{c|c}
\hline \hline$C_{11}^{R}$ & $\frac{1}{2 M} \frac{A_{r} Z^{2} e^{2} \rho}{(4 \rho+r)}\left(\frac{40 \rho^{2}+7 \rho r+r^{2}}{\rho^{2} r^{4}}\right)$ \\
$C_{12}^{R}$ & $\frac{A_{r} Z^{2} e^{2}}{r^{3} M(4 \rho+r)}+\frac{4 A_{r} Z^{2} e^{2} \rho}{r^{4} M(4 \rho+r)}$ \\
$C_{44}^{R}$ & $-\frac{A_{r} Z^{2} e^{2}}{r^{3} M(4 \rho+r)}-\frac{4 A_{r} Z^{2} e^{2} \rho}{r^{4} M(4 \rho+r)}$
\end{tabular}

TABLE V

Values of lattice sum for Coulomb potential. The units of $C_{i j}$ are $Z^{2} e^{2} / \Delta a$, where $\Delta$ is the volume of the cell.

\begin{tabular}{l|c}
\hline \hline$A_{a}$ & 1.74756 \\
$A_{r}$ & 1.74756 \\
$C_{11}^{e}$ & -2.55604 \\
$C_{12}^{e}$ & 0.11298 \\
$C_{44}^{e}$ & 1.27802
\end{tabular}

TABLE VI

Calculated values of SOEC and experimental values.

\begin{tabular}{c|c|c|c|c|c|c}
\hline \hline & $\begin{array}{c}C_{11} \times 10^{11} \\
{\left[\mathrm{dyn} / \mathrm{cm}^{2}\right]} \\
\text { Present }\end{array}$ & $\begin{array}{c}C_{11} \times 10^{11} \\
{\left[\mathrm{dyn} / \mathrm{cm}^{2}\right]} \\
\text { Exp. [12] }\end{array}$ & $\begin{array}{c}C_{12} \times 10^{11} \\
{\left[\mathrm{dyn} / \mathrm{cm}^{2}\right]} \\
\text { Present }\end{array}$ & $\begin{array}{c}C_{12} \times 10^{11} \\
{\left[\mathrm{dyn} / \mathrm{cm}^{2}\right]} \\
\text { Exp. [12] }\end{array}$ & $\begin{array}{c}C_{44} \times 10^{11} \\
{\left[\mathrm{dyn} / \mathrm{cm}^{2}\right]} \\
\text { Present }\end{array}$ & $\begin{array}{c}C_{44} \times 10^{11} \\
{\left[\mathrm{dyn} / \mathrm{cm}^{2}\right]} \\
\text { Exp. }[12]\end{array}$ \\
\hline $\mathrm{NaCl}$ & 4.74 & 4.79 & 1.29 & 1.15 & 1.27 & 1.27 \\
$\mathrm{KCl}$ & 3.75 & 3.98 & 0.79 & 0.61 & 0.81 & 0.63
\end{tabular}

\section{Results and discussion}

In this work, the applicability of the interatomic potential is considered for studying the crystalline properties of alkali halides. This potential form represents the composite form of the inverse power dependence and the exponential dependence of the repulsive energy. Second order elastic constants are calculated using the same potential and compared with the experimental data and some other theoretical works.

Figure 1a,b and Fig. 2a,b show that $\Delta G$ tends to 0 at the phase transition pressure 28.69 $\mathrm{Gpa}$ for $\mathrm{NaCl}$ and 2.4 Gpa for $\mathrm{KCl}$ crystals, respectively. These values of the phase transition pressures are in much better agreement with the available experimental results $[2,5,15]$.

We have also calculated the percentage of relative volume changes using the compression curve and presented in Fig. 1a,b and Fig. 2a,b. The values of these 
volume collapses $\Delta V(P) / V(0)$ have been obtained at the phase transition pressures and listed in Table VII along with their available experimental data $[2,16]$.
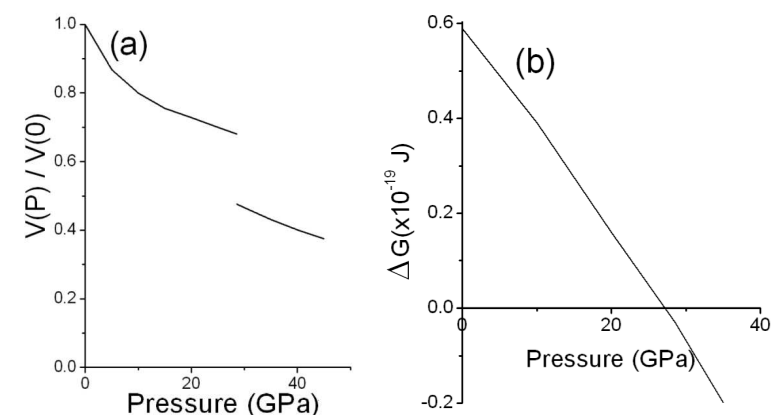

Fig. 1. $\mathrm{NaCl}$ : variation in relative volume $V(P) / V(0)$ with pressure $P($ a) and in Gibbs free energy difference $\Delta G$ with pressure $P(\mathrm{~b})$.
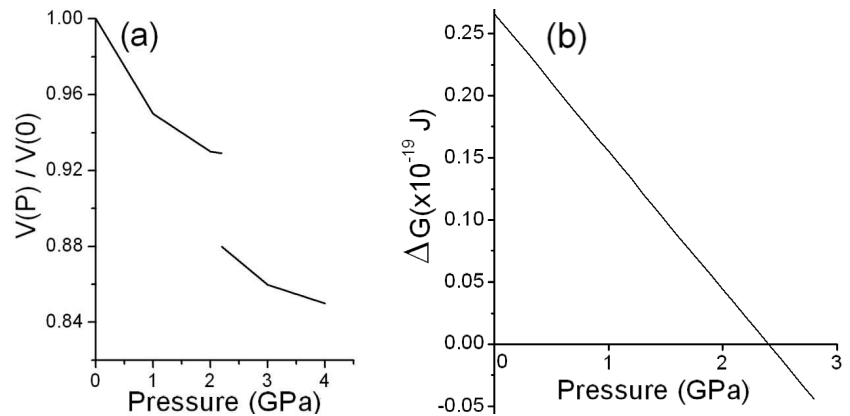

Fig. 2. $\mathrm{KCl}$ : variation in relative volume $V(P) / V(0)$ with pressure $P(\mathrm{a})$ and in Gibbs free energy difference $\Delta G$ with pressure $P(\mathrm{~b})$.

TABLE VII

Phase transition pressures and volume collapses of alkali halides.

\begin{tabular}{c|c|c|c|c|c}
\hline \hline \multirow{2}{*}{ Crystal } & \multicolumn{3}{|c|}{$\begin{array}{c}\text { Phase transition } \\
\text { pressure [GPa] }\end{array}$} & \multicolumn{2}{c}{$\begin{array}{c}\text { Volume collapses } \\
\end{array}$} \\
\cline { 2 - 6 } $\mathrm{NaCl}$ & Present & Experiment & Others & Present & Experiment \\
& 28.6 & $29.0^{a}$ & $27.0^{b}$ & -4.5 & $-5.8^{e}$ \\
& & & $27.4^{c}$ & & \\
& & & $23.9^{d}$ & & $-12.3^{f}$ \\
$\mathrm{KCl}$ & 2.4 & $2.0^{f}$ & $1.1^{b}$ & -14.3 & \\
& & $1.9^{g}$ & & & \\
\hline
\end{tabular}

${ }^{a}$ Ref. [15], ${ }^{b}$ Ref. [17], ${ }^{c}$ Ref. [18], ${ }^{d}$ Ref. [19], ${ }^{e}$ Ref. [18], ${ }^{f}$ Ref. [2], ${ }^{g}$ Ref. [5]. 
The results which are presented in Table VII reveal that the phase transition pressure and the associated volume collapses are, generally, in good agreement with the available experimental results better than those obtained by other theoretical workers.

On the basis of overall achievements, it may be concluded that the effective pair potential model approach adopted by us is capable of providing a better understanding of the high-pressure phase transition behavior of alkali halides.

\section{References}

[1] R.K. Singh, Phys. Rep. 85, 259 (1982).

[2] P.W. Bridgman, Proc. Am. Acad. Arts. Sci. 76, 9 (1945).

[3] H.R. Yazar, S. Ağan, K. Çolakoğlu, Turk J. Phys. 25, 345 (2001).

[4] M.L. Cohen, S. Froyen, Phys. Rev. B 29, 3770 (1984).

[5] G.C. Kennedy, S.N. Vaidya, J. Phys. Chem. Solids 32, 951 (1971).

[6] V.V.S. Nirwal, R.K. Singh, Phys. Rev. B 20, 5379 (1979).

[7] K.N. Jog, R.K. Singh, S.P. Sanyal, Phys. Rev. B 31, 6047 (1985).

[8] D.B. Sirdeshmukh, K.G. Subhadra, Phys. Status Solidi B 150, K11 (1988).

[9] J. Michielson, P. Woerlee, F.V.D. Graff, A.A. Ketelaar, J. Chem. Soc. Faraday Trans. II 71, 1730 (1975).

[10] D.W. Hafemeister, W.H. Flygare, J. Chem. Phys. 43, 795 (1965).

[11] W. Cochran, Solid State Sci. 21, 63 (1971).

[12] C.S. Smith, L.S. Chain, J. Phys. Chem. Solids 36, 205 (1975).

[13] L. Orson, C. Anderson, R.W. Robert, Phys. Earth Planet, Interiors 3, North Holland, Amsterdam 1975, p. 63.

[14] O.L. Anderson, H. Oda, Rev. Geophys. 30, 57 (1992).

[15] D.L. Heinz, R. Jeanloz, Phys. Rev. B 30, 6045 (1984).

[16] W.A. Basset, T. Takahashi, H.K. Mao, J.S. Weaver, J. Appl. Phys. 39, 319 (1968).

[17] M.L. Cohen, S. Froyen, J. Phys. C, Solid State Phys. 19, 2623 (1986).

[18] R.K. Singh, N.V.K. Prabhakar, Phys. Status Solidi B 141, K29 (1987).

[19] K.N. Jog, PhD. Thesis, Rani Durgawati University, Jabalpur 1988, p. 77. 